\title{
Contemporary management of spinal AVFs and AVMs: lessons learned from 110 cases
}

\author{
*Leonardo Rangel-Castilla, M.D., Jonathan J. Russin, M.D., Hasan A. Zaidi, M.D., \\ Eduardo Martinez-del-Campo, M.D., Min S. Park, M.D., Felipe C. Albuquerque, M.D., \\ Cameron G. McDougall, M.D., Peter Nakaji, M.D., and Robert F. Spetzler, M.D.
}

Division of Neurological Surgery, Barrow Neurological Institute, St. Joseph's Hospital and Medical Center, Phoenix, Arizona

\begin{abstract}
Object. Spinal arteriovenous fistulas (AVFs) and arteriovenous malformations (AVMs) are rare, complex spinal vascular lesions that are challenging to manage. Recently, understanding of these lesions has increased thanks to neuroimaging technology. Published reports of surgical results and clinical outcome are limited to small series. The authors present a large contemporary series of patients with spinal AVFs and AVMs who were treated at Barrow Neurological Institute in Phoenix, Arizona.

Methods. Retrospective detailed review of a prospective vascular database was performed for all patients with spinal AVFs and AVMs treated between 2000 and 2013. Patient demographic data, AVF and AVM characteristics, surgical results, clinical outcomes, complications, and long-term follow-up were reviewed.

Results. Between 2000 and 2013, 110 patients (57 male and 53 female) underwent obliteration of spinal AVFs and AVMs. The mean age at presentation was 42.3 years (range 18 months-81 years). There were 44 patients with AVFs and 66 with AVMs. The AVM group included 27 intramedullary, 21 conus medullaris, 12 metameric, and 6 extradural. The most common location was thoracic spine (61\%), followed by cervical (22.7\%), lumbar (14.5\%), and sacral (1.8\%). The most common presenting signs and symptoms included paresis/paralysis (75.5\%), paresthesias (60\%), pain (51.8\%), bowel/bladder dysfunction (41.8\%), and myelopathy (36.4\%). Evidence of rupture was seen in $26.4 \%$ of patients. Perioperative embolization was performed in $42 \%$ of patients. Resection was performed in 95 patients $(86.4 \%)$. Embolization alone was the only treatment in 14 patients $(12.7 \%)$. One patient was treated with radiosurgery alone. Angiographically verified AVF and AVM obliteration was achieved in 92 patients $(83.6 \%)$. At a mean follow-up duration of 30.5 months (range 1-205 months), 43 patients (97.7\%) with AVFs and 57 (86.4\%) with AVMs remained functionally independent (McCormick Scale scores $\leq 2$ ). Perioperative complications were seen in 8 patients (7\%). No deaths occurred. Temporary neurological deficits were observed in 27 patients $(24.5 \%)$. These temporary deficits recovered 6-8 weeks after treatment. Recurrence was identified in 6 patients (13.6\%) with AVFs and $10(15.2 \%)$ with AVMs.

Conclusions. Spinal AVFs and AVMs are complex lesions that should be considered for surgical obliteration. Over the last several decades the authors have changed surgical strategies and management to achieve better clinical outcomes. Transient neurological deficit postoperatively is a risk associated with intervention; however, clinical outcomes appear to exceed the natural history based on patients' ability to recover during the follow-up period. Due to the recurrence rate associated with these lesions, long-term follow-up is required.
\end{abstract}

(http://thejns.org/doi/abs/10.3171/2014.7.FOCUS14236)

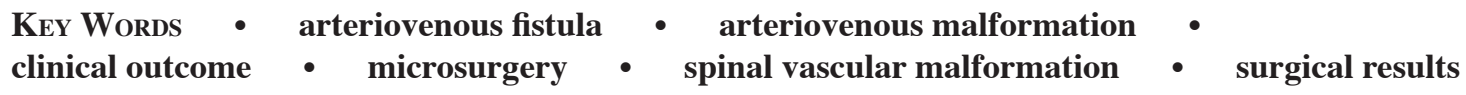

$\mathrm{S}$ PINAL arteriovenous fistulas (AVFs) and arteriovenous malformations (AVMs) are complex neurosurgical lesions that are very challenging to manage. Spinal vascular malformations account for $3 \%-4 \%$ of all intradural spinal cord mass lesions. ${ }^{2}$ Over the last few decades our understanding of these lesions has dramatically increased thanks to neuroimaging technology (e.g., spinal

\footnotetext{
Abbreviations used in this paper: $\mathrm{AVF}=$ arteriovenous fistula; $\mathrm{AVM}=$ arteriovenous malformation; $\mathrm{ICG}=$ indocyanine green; SAH $=$ subarachnoid hemorrhage.

* Drs. Rangel-Castilla and Russin contributed equally to this work.
}

angiography and indocyanine green [ICG] angiography) and the availability of specialized neurovascular centers. ${ }^{2,4,5,9,10,15,18-21}$ Based on specific anatomical locations and pathophysiological factors, spinal AVFs are classified as extradural, intradural dorsal, and intradural ventral. Similarly, spinal AVMs are classified as intramedullary, conus medullaris, metameric, and extradural. ${ }^{10,15,18}$ Various treatment modalities including conservative observation, endovascular embolization, microsurgical resection, radiation therapy, and combined therapies have been reported. Due to the rarity of these spinal vascular lesions, reports of their management and outcomes have been limited to small series and case reports. , $, 5,8,9,18-20,22^{2}$ 
The purpose of this work is to present the postoperative surgical results and the long-term clinical outcomes for a large contemporary series of patients with spinal AVFs and AVMs from a neurovascular tertiary referral center.

\section{Methods}

The prospectively maintained vascular database from Barrow Neurological Institute (Phoenix, Arizona) was reviewed. All patients with a spinal AVF or AVM who underwent microsurgical treatment between June 2000 and December 2013 were included in the analysis. Patient demographic data, medical comorbidities, presenting signs and symptoms, baseline neurological examinations, postoperative examinations, and outcome at the last clinical follow-up were obtained. Pretreatment, postoperative, and follow-up imaging was evaluated by the senior authors (P.N. and R.F.S.) and endovascular neurosurgeons (F.C.A. and C.G.M.). Functional status was measured using the McCormick Scale preoperatively, postoperatively, and at last clinical follow-up.

\section{Results}

\section{Patient and AVF/AVM Characteristics}

During the study period, spinal AVFs and AVMs were managed surgically in 110 patients. Fifty-seven $(51.8 \%)$ were male and $53(48.2 \%)$ were female, with a mean age of 42.3 years (range 18 months-81 years). Of those 110 patients, $44(40 \%)$ had AVFs and $66(60 \%)$ had AVMs. The AVM group included 27 (40.9\%) intramedullary, $21(31.8 \%)$ conus medullaris, $12(18.2 \%)$ metameric, and $6(9.1 \%)$ extradural lesions. The vascular malformations were most common in the thoracic region $(61 \%)$, followed by the cervical (22.7\%), lumbar (14.5\%), and sacral $(1.8 \%)$ spine.

\section{Presentation and Radiographic Findings}

Table 1 summarizes the clinical presentation of patients with spinal AVFs and AVMs. The most common presentation was paresis/paralysis in 83 patients $(75.5 \%)$, and 20 patients $(18.2 \%)$ were asymptomatic. Other symptoms included pain in $57(51.8 \%)$, paresthesias in $66(60 \%)$, bowel and/or bladder dysfunction in 46 (41.8\%), and myelopathy in $40(36.4 \%)$. Twenty-nine patients $(26.4 \%)$ had radiographic evidence of rupture, more commonly with AVMs than AVFs. Hemorrhage was most common in intramedullary types (52\%). One asymptomatic pediatric patient was diagnosed after an abdominal murmur/thrill was discovered. One patient with a conus medullaris AVM presented initially with cerebral subarachnoid hemorrhage (SAH). One patient was misdiagnosed with a spinal cord tumor and taken to surgery for resection at another hospital. Surgery was aborted and the patient was transferred to our institution for definitive care. Spinal cord edema was seen in $88(80 \%)$ of the patients. Other radiographic findings included a syrinx in 15 patients $(13.6 \%)$, and a nidusassociated aneurysm in 12 patients $(10.9 \%)$.

\section{Management of the Lesions}

Of the 110 patients, 34 (30.9\%) had undergone prior

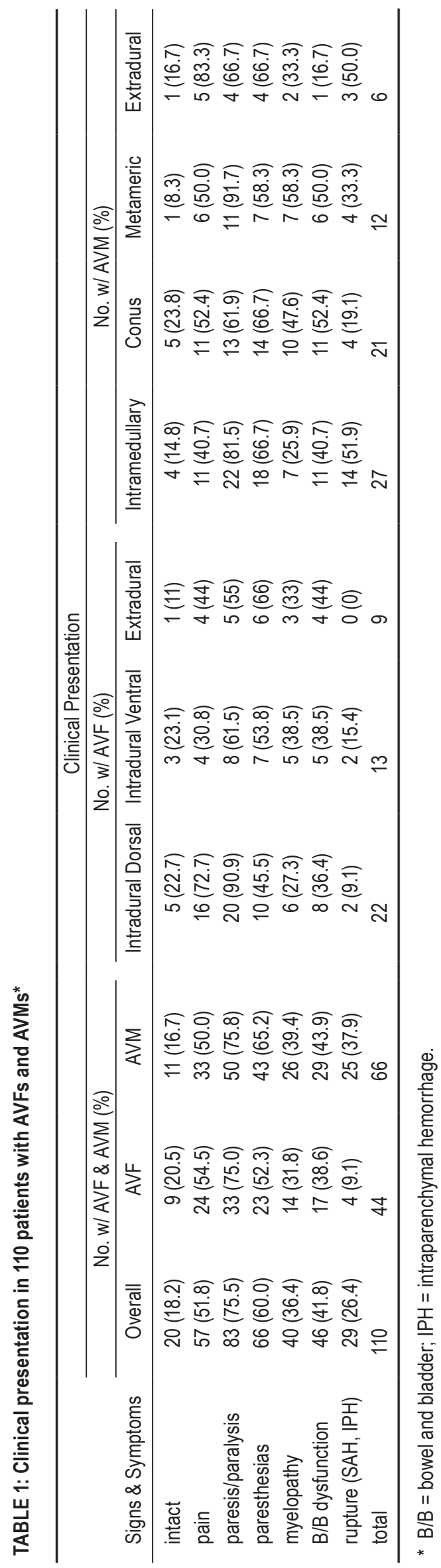

Neurosurg Focus / Volume 37 / September 2014 


\section{Contemporary surgical management of spinal AVFs and AVMs}

therapy before they presented to Barrow Neurological Institute; $8(18.2 \%)$ patients with AVFs and 26 (39.4\%) patients with AVMs. Previous treatment attempts included 4 (3.6\%) resections, $16(14.5 \%)$ endovascular embolizations, $12(10.9 \%)$ surgeries with embolization, and $2(1.8 \%)$ radiation treatments.

\section{Microsurgical Resection}

Ninety-five patients $(86.4 \%)$ underwent microsurgical resection. Typically, we use a multilevel laminoplasty extending a single level above and below the AVM nidus or the AVF shunt. As described previously, ${ }^{9,19,22}$ laminoplasty is preferred over laminectomy to protect the underlying neural elements and to facilitate future operations. If necessary, the laminotomy is extended to the ipsilateral pedicle to improve lateral exposure. Similarly, the dural opening is tailored to the lesion's location. Dentate ligaments are routinely sectioned for further anterolateral exposure if necessary. At times, dorsal nerve rootlets are sacrificed to improve exposure.

For spinal AVFs, the abnormal arteriovenous connection is carefully identified preoperatively on the spinal angiogram. Since May 2005, intraoperative ICG angiography has been used to assist in identifying the anomalous arteriovenous connection. This abnormal arteriovenous connection is obliterated with an aneurysm clip and/ or coagulated and sectioned. For spinal AVMs, a more careful and complete inspection of the spinal cord and its associated vasculature is necessary to achieve optimal surgical results. It is often difficult to clearly define the angioarchitecture of these complex lesions. Intraoperative ICG angiography has been helpful in identifying feeding arterial pedicles and early draining veins (Fig. 1). Repeated ICG videoangiography at various time points during the AVM resection can facilitate identification of residual, early-filling pedicles that are then obliterated. Embolic material casts from endovascular embolization also help to identify associated feeding arteries, allowing correlation of the surgical anatomy with the AVM angioarchitecture (Figs. 1-3).

Currently in our practice, myelotomy is reserved for AVMs in which the nidus is completely intraparenchymal. Other indications for myelotomy include fenestration of an associated syrinx, or evacuation of an intraparenchymal hematoma. Four classic myelotomies are described. These include a midline dorsal, dorsal root entry zone, lateral myelotomy, and anterior midline myelotomy. However, most surgical interventions for spinal AVFs or AVMs do not require a myelotomy. The pial resection technique is currently used for the majority of the spinal AVMs we treat. ${ }^{19}$ In this technique, feeding arteries and draining veins are coagulated and divided along the spinal cord surface while minimizing subpial dissection (Figs. 1-3). Interruption of spinal AVMs at the pial surface provides sufficient devascularization to alleviate venous hypertension. Endovascular embolization is an integral part in the surgical management of spinal AVFs and AVMs, having diagnostic as well as therapeutic applications. Embolization was used in 40 (42\%) of the 95 patients who underwent microsurgical resection. Embolization alone was the only treatment in 14 (12.7\%) of the
110 patients. One patient with an intramedullary AVM was treated with radiosurgery alone.

\section{Clinical Outcomes}

Clinical outcomes for the 110 patients were assessed using the McCormick functional classification scale (Table 2) at presentation to our institution, postoperatively, and at the time of latest available clinical follow-up. The mean clinical and radiographic follow-up time was 30.5 months (range 1-205 months). The mean McCormick Scale score at initial presentation was $2.7 \pm 1.1$ for spinal AVFs and $2.5 \pm 0.9$ for spinal AVMs. The immediate postoperative mean McCormick Scale score was $2.2 \pm$ 1.0 for spinal AVFs and $2.7 \pm 1.0$ for spinal AVMs. At the latest follow-up the mean McCormick Scale score was $1.8 \pm 1.0$ for spinal AVFs and $2.2 \pm 1.0$ for spinal AVMs. When preoperative status is compared with latest followup there is a statistically significant improvement in patients treated for spinal AVFs and AVMs. The McCormick Scale score for patients with spinal AVFs improved by 0.9 , and for patients with spinal AVMs it improved by 0.3 ; both were statistically significant $(\mathrm{p}<0.01$ and $\mathrm{p}=$ 0.01 , respectively).

\section{Temporary Neurological Decline}

Of the 20 patients who were neurologically intact at the time of initial presentation, 4 had temporary paresthesias postoperatively and 16 remained intact. Of the 90 patients with a preoperative neurological deficit, 24 patients $(26.7 \%)$ regained almost full neurological function postoperatively. These 24 patients also had associated intraparenchymal or subdural hematomas, a syrinx, and/or a tethered spinal cord additionally treated at the time of surgery. Significant function decline was observed postoperatively in 3 patients $(6.8 \%)$ in the AVF group and in 20 patients $(30.3 \%)$ in the AVM group. At last follow-up only 1 patient $(2.3 \%)$ from the AVF group and 9 patients (13.6\%) in the AVM group had persistent neurological deficits. At last follow-up, $25(71.4 \%)$ of 35 patients with spinal AVFs and 24 (43.6\%) of 55 patients with AVMs who had a preoperative neurological deficit showed neurological improvement. Similarly, subdividing the AVM group into the 4 different types, we observed a neurological improvement in $8(34.8 \%)$ of 23 in the intramedullary group, $11(68.7 \%)$ of 16 in the conus medullaris, 4 (36.4\%) of 11 in the metameric, and $1(20 \%)$ of 5 in the extradural group. Overall, a good outcome, defined as unchanged or improved functional status compared with preoperative examination, was seen in 43 patients $(97.7 \%)$ with spinal AVFs and in $57(86.4 \%)$ with spinal AVMs. There were no deaths in either of the groups.

Four patients suffered iatrogenic vertebral artery injury during embolization. Of these 4 patients, 2 had retained microcatheters, one of whom developed paresthesias in the lower extremities, and the other of whom suffered an anterior spinal cord infarct and quadriplegia. The other 2 patients with vertebral artery injuries had perforations of a major vertebral artery branch requiring parent vessel occlusion. The first of these patients had no neurological or clinical sequelae, and the second had an 

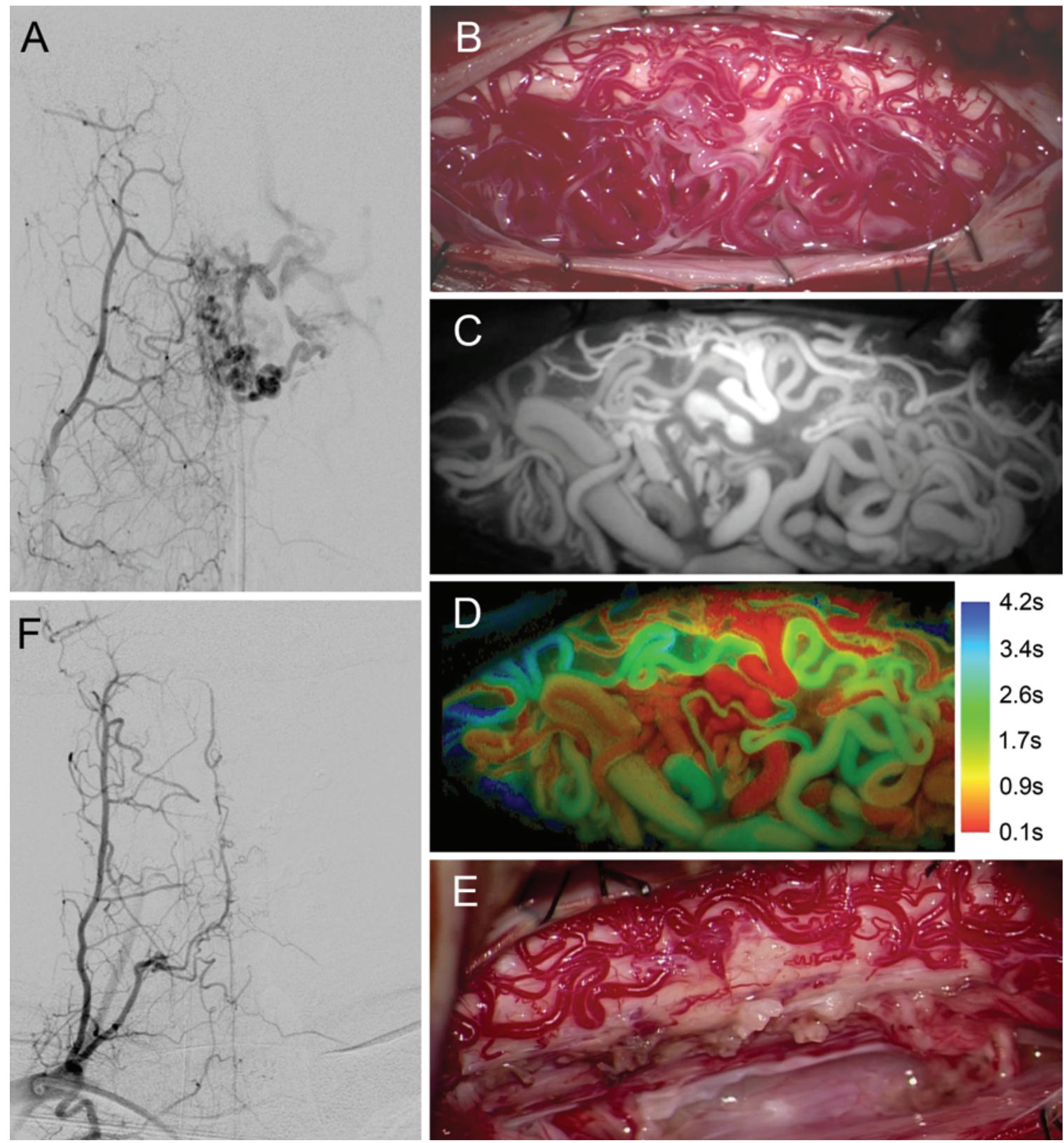

FIG. 1. Cervical spinal AVM treated in a 26-year-old woman with 2 previous bleeding episodes and left hemiparesis with complete recovery. A: Preoperative spinal angiography showing the cervical AVM feeding from the ascending pharyngeal and vertebral arteries (not shown). B: Intraoperative microscope view of the malformation before resection. C: Intraoperative ICG angiography demonstrating the angioarchitecture of the malformation. D: Flow 800 image showing different flow velocities within the malformation. E: Intraoperative microscope view of the spinal cord after surgery was performed using the pial resection technique. F: Postoperative spinal angiogram showing gross-total AVM resection. Note the preservation of the anterior spinal artery.

anterior spinal artery rupture with SAH and hydrocephalus requiring ventriculoperitoneal shunt placement. Two patients with intramedullary AVMs had symptomatic postoperative intraparenchymal hemorrhage requiring surgical evacuation. One patient with a lesion in the conus medullaris developed bowel and bladder incontinence after embolization. One patient with a conus medullaris AVM developed a postoperative epidural hematoma, which was managed conservatively. One patient with a metameric AVM developed incomplete Brown-Séquard syndrome after surgery. Other procedures required were ventriculoperitoneal shunt placement to treat hydrocephalus in 3 patients, CSF leak repair in 3 patients, and wound exploration for infection in 5 patients. At the latest followup, 8 patients required surgery for spinal cord tethering.

\section{Angiographic Results and Long-Term Radiographic Follow-Up}

Postoperative spinal angiography was performed in all patients within $24-48$ hours after surgery. Total oblit- 


\section{Contemporary surgical management of spinal AVFs and AVMs}
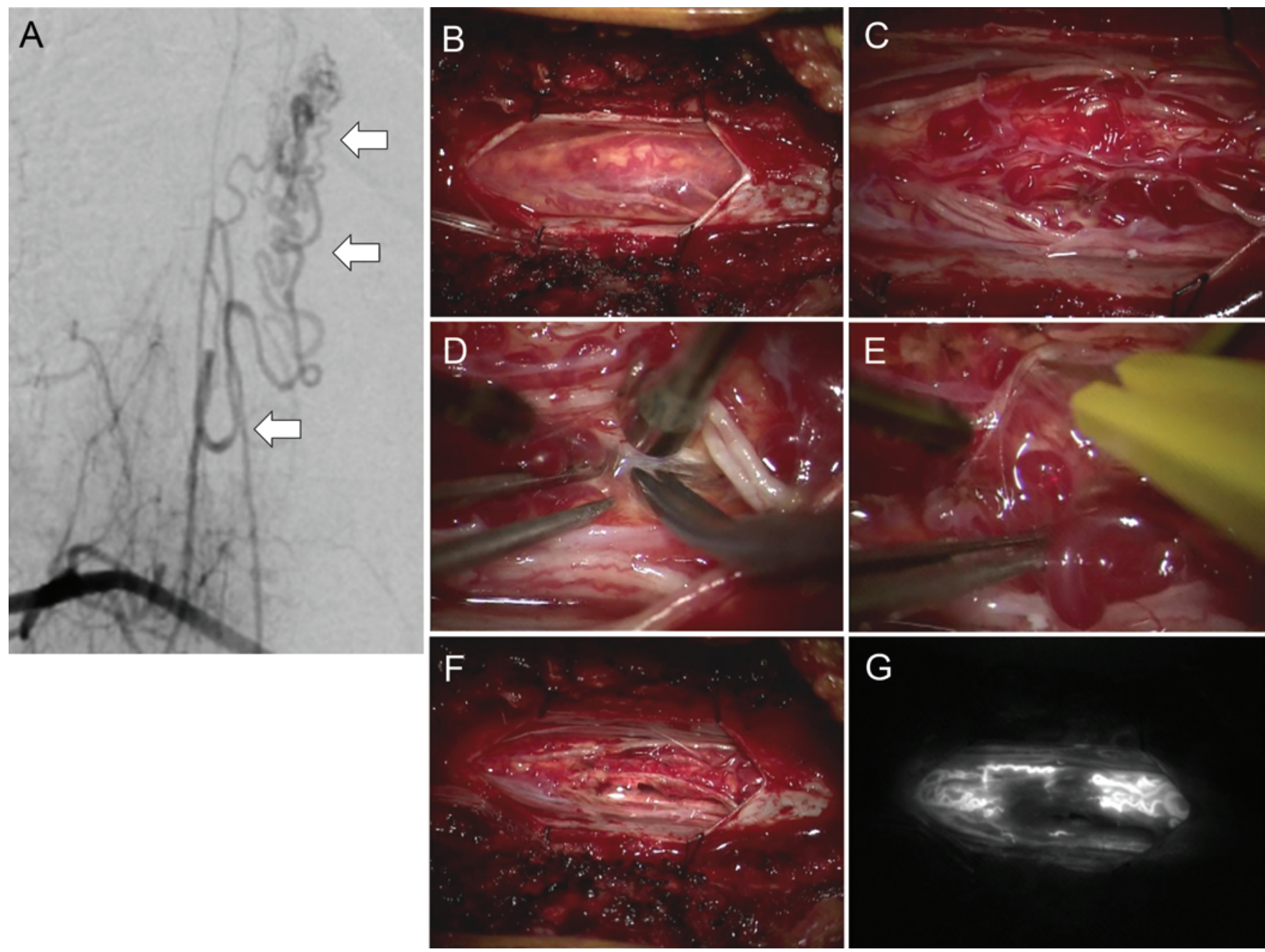

FIG. 2. Thoracic Type 2 spinal AVM treated in a 32-year-old otherwise healthy man who presented with sudden-onset back pain and complete paraplegia. Selective right T-8 anteroposterior spinal angiogram (A) was notable for a Type 2 spinal AVM (arrows). Patient underwent a thoracic laminoplasty (B) with intraoperative evidence of a large partially hemorrhagic AVM (C). A myelotomy was performed adjacent to the nidus and a large intramedullary hematoma was evacuated (D). The nidus was circumferentially dissected and feeding vessels $(\mathrm{E})$ were carefully identified, sharply cut, and mobilized. The postresection cavity (F) and ICG angiography (G) showed evidence of hyperemic vessels with complete resection of the AVM nidus. At long-term follow-up the patient had some improved sensation but did not regain motor function.

eration was reported in $92(83.6 \%)$ of the 110 patients: $42(95.5 \%)$ of the 44 patients with spinal AVFs and 50 (75.7\%) of the 66 patients with spinal AVMs.

Recurrence was identified in 6 patients $(13.6 \%)$ in the AVF group and in 10 patients $(15.2 \%)$ in the AVM group. The 6 recurrent AVFs presented 7-48 months after treatment, and all patients underwent retreatment except for one who refused. Of the 10 AVM recurrences, 6 were intramedullary, 3 were conus medullaris, and 1 was an extradural lesion. These recurrences were diagnosed between 18 and 108 months after initial treatment. Retreatment was recommended for all spinal AVM recurrences; however, it was performed in only 6 patients.

\section{Discussion}

Over the years a gradual paradigm shift in the understanding, classification, and management of spinal AVMs has occurred. As spinal angiography techniques evolved, radiographic imaging of spinal AVFs and AVMs afforded the opportunity to better understand the pathophysiological mechanisms, facilitating the development of an anato-

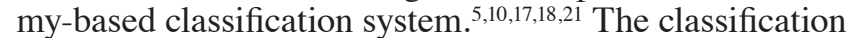
proposed by Kim and Spetzler represents an evolution that incorporates the enhanced understanding of these entities in recent decades. ${ }^{10}$ Their classification system is based on the anatomical location of each lesion, with the corresponding pathophysiological mechanism. This study represents the most contemporary series of spinal AVFs and AVMs treated at a tertiary referral neurovascular center. Similarly to previous reports of patients with spinal vascular malformations, most of the patients in the current series presented with a history of stepwise progressive neurological deterioration. ${ }^{3,5,9,11,15,19,22}$ Overall, $75 \%$ of our patients presented with insidious paresis/ paralysis. Twenty-nine (26.4\%) had evidence of rupture, 

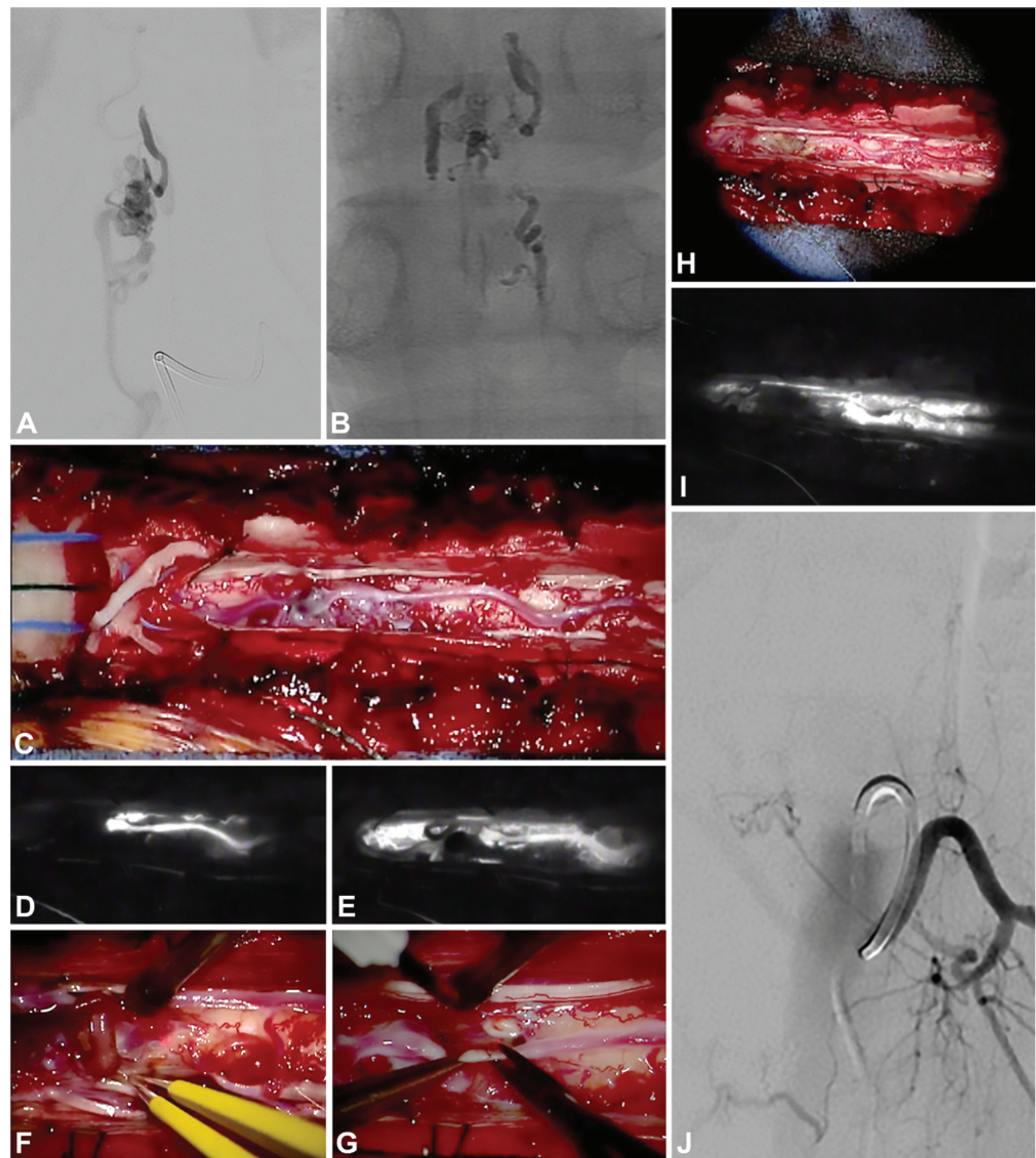

FIG. 3. Thoracolumbar Type 2 AVM treated in a 17-year-old otherwise healthy boy who presented with sudden-onset paraparesis. A selective left T-11 anteroposterior angiogram (A) showing a Type 2 thoracic spinal AVM that was then embolized with glue, with incomplete obliteration (B). A thoracic laminoplasty was performed (C) and was notable for a large spinal AVM. Early (D) and late (E) ICG angiography was notable for early venous filling. The nidus was circumferentially dissected (F) and sharply dissected (G) off the spinal cord, with postoperative cavity (H) and ICG angiography (I) demonstrating only hyperemic vessels. Postoperative angiography $(\mathrm{J})$ confirmed complete AVM obliteration. At long-term follow-up the patient regained motor function with ability to ambulate with a walker, but with persistent genitourinary dysfunction. 


\section{Contemporary surgical management of spinal AVFs and AVMs}

TABLE 2: Clinical outcomes in 110 patients with AVFs or AVMs

\begin{tabular}{lcccccc}
\hline & & \multicolumn{3}{c}{ Mean McCormick Score } & & \\
\cline { 3 - 5 } \multicolumn{1}{c}{ Outcome } & No. of Cases & Preop & Postop & Last Follow-Up & Improvement & $p$ Value \\
\hline overall & 110 & $2.6 \pm 1.0$ & $2.5 \pm 1.0$ & $2.1 \pm 1.0$ & 0.5 & $<0.01$ \\
AVFs & 44 & $2.7 \pm 1.1$ & $2.2 \pm 1.0$ & $1.8 \pm 1.0$ & 0.9 & $<0.01$ \\
$\quad$ intradural dorsal & 22 & $2.5 \pm 0.9$ & $2.7 \pm 1.0$ & $2.0 \pm 0.9$ & 0.5 & 0.02 \\
intradural ventral & 13 & $2.7 \pm 1.0$ & $2.9 \pm 1.0$ & $2.0 \pm 1.0$ & 0.7 & $<0.01$ \\
$\quad$ extradural & 9 & $1.9 \pm 1.1$ & $2.0 \pm 0.9$ & $1.7 \pm 1.0$ & 0.2 & 0.05 \\
AVMs & 66 & $2.5 \pm 0.9$ & $2.7 \pm 1.0$ & $2.2 \pm 1.0$ & 0.3 & 0.01 \\
intramedullary & 27 & $2.4 \pm 0.9$ & $2.6 \pm 1.0$ & $2.3 \pm 1.1$ & 0.1 & 0.6 \\
conus medullaris & 21 & $2.3 \pm 0.9$ & $2.3 \pm 1.1$ & $1.9 \pm 0.9$ & 0.4 & 0.04 \\
metameric & 12 & $3.0 \pm 0.9$ & $3.2 \pm 0.7$ & $2.6 \pm 0.9$ & 0.4 & 0.05 \\
extradural & 6 & $2.5 \pm 1.0$ & $2.8 \pm 1.2$ & $2.5 \pm 1.0$ & 0 & 1 \\
\hline
\end{tabular}

more commonly in AVMs than AVFs. Intramedullary and extradural AVMs had the highest rupture rates. ${ }^{8,14}$

\section{Management of the Lesions}

The management of spinal vascular malformations depends on the type and location of the lesion as well as the surgical experience and capabilities of the practitioners in the neurosurgical center. Conservative management has gradually been replaced by endovascular embolization and/or resection. . $^{2-5,8,9,11-13,15,16,18,19,21,22}$ At our institution, we advocate preoperative endovascular embolization followed by microsurgical obliteration or resection in the majority of cases., ${ }^{9,10,18,19,22}$ However, endovascular embolization is not possible for all cases of spinal vascular malformations. In the current series, preoperative embolization was used in $42 \%$ of patients who underwent surgery; $19.4 \%$ in the AVF and and $56 \%$ in the AVM group. Because of their complex angioarchitecture when compared to spinal AVFs, AVMs underwent preoperative embolization more frequently. Endovascular embolization was the only treatment in $12.7 \%$ of the cases overall (18.2\% of the AVFs and $9.1 \%$ of the AVMs).

As highlighted previously, embolization of spinal AVMs can be particularly challenging, especially for intramedullary and conus medullaris lesions. Given the eloquence of surrounding tissues and the complexity of spinal AVMs, obliteration by endovascular means is challenging. Complete endovascular angiographic occlusion of selected cases of spinal AVFs and AVMs has been reported, with variable outcomes. ${ }^{3-5,7,9,11-13,15,19,22}$ With a multimodality approach used in these 110 cases, the rate of immediate complete angiographic obliteration was 83.6\%: $95.5 \%$ for AVFs and 75.7\% for AVMs. These results are similar to other series of spinal AVFs and AVMs, including previous reports from our institution. Wilson et al. ${ }^{22}$ reported an $88 \%$ obliteration rate for conus medullaris AVMs, Boström et al. ${ }^{3}$ had a $78.5 \%$ obliteration rate for spinal glomus-type AVMs, and Kalani et al. ${ }^{9}$ reported an $89 \%$ obliteration rate for pediatric spinal AVMs.

\section{Transient Neurological Deficit}

Using the McCormick functional scale score we report the rate of transient neurological deterioration ob- served postoperatively. There were 20 patients who were totally asymptomatic prior to surgery. Of those 20 patients, 4 had transitory neurological dysfunction postoperatively. Ninety patients initially presented with a neurological deficit and $26.7 \%$ showed improvement immediately after surgery. This improvement was probably related to the decompression of AVM mass effect, intraparenchymal or subdural hematoma evacuation, syrinx drainage, and/or detethering of the spinal cord. Neurological decline was observed in $6.8 \%$ of the patients with AVFs and in 30.3\% of the patients with AVMs. This finding is consistent with previously published series, in which the rate of transient neurological decline ranged from $25 \%$ to $38 \%$. In the current study, at a mean follow-up of 30.5 months, $71.4 \%$ of patients with AVFs and $43.6 \%$ of patients with AVMs who had postoperative neurological dysfunction showed improvement. $5,9,19,22$ Of the patients with AVMs, the conus medullaris group showed the most neurological recovery (68.7\%), followed by the metameric (36.4\%), intramedullary (34.8\%), and extradural (20\%) groups. Overall, at the latest follow-up we observed good to excellent outcomes in $97.7 \%$ and $86.4 \%$ of the patients with spinal AVFs and AVMs, respectively. We have found this information to be very valuable for patients when counseling them preoperatively. These results are consistent with other recent contemporary series. Reported good outcomes after treatment for spinal AVMs varies from $70 \%$ to $95 \%$ in the current literature. ${ }^{3,9,19,22}$

The risk of treating spinal AVFs and AVMs must be weighed against the natural history of these lesions. Data on natural history are sparse; however, there is consensus about the unremitting progression of neurological decline for untreated spinal AVFs and AVMs. Approximately 50\% of patients with untreated spinal AVMs are severely disabled within 3 years and less than $10 \%$ are able to ambulate without assistance. ${ }^{1}$ Other investigators have reported a severe disability at 20 years if patients with spinal AVMs are left untreated. ${ }^{6}$ In our series, although $6.8 \%$ of patients with AVFs and $30.3 \%$ of patients with AVMs had a transient neurological decline postoperatively, we found good to excellent outcome at latest follow-up in $97.7 \%$ and $86.4 \%$ of the patients with AVFs and AVMs, respectively.

Microsurgical techniques for spinal AVM resection 


\section{Rangel-Castilla et al.}

have improved over the past few decades. Traditional myelotomy is not as frequently used as before, and its use is typically limited to intramedullary lesions with intraparenchymal hematoma or syrinx. The senior author has pioneered the pial resection technique for spinal AVM resection, with satisfactory clinical and radiographic results. ${ }^{19}$ Occluding the AVM at the pial border rather than following the nidus into the spinal cord parenchyma aims to reduce tissue dissection while obliterating the lesion.

Major limitations of our study include its retrospective nature and the incomplete long-term angiographic follow-up. A common problem for tertiary referral institutions is the follow-up in patients from other cities or countries.

\section{Conclusions}

Spinal AVFs and AVMs are complex lesions that should be considered for surgical obliteration. Over the last decades we have changed our surgical strategies and management to achieve better clinical outcomes. Transient neurological deficit postoperatively is a risk of intervention; however, clinical outcomes appear to exceed the natural history based on the ability of patients to recover during the follow-up period. Due to the recurrence rate identified in these lesions, they require long-term followup.

\section{Disclosure}

Dr. McDougall is a consultant for Terumo (MicroVention) and Covidien (ev3).

Author contributions to the study and manuscript preparation include the following. Conception and design: Nakaji, RangelCastilla, Russin. Acquisition of data: Rangel-Castilla, Russin, Zaidi, Martinez-del-Campo, Park. Analysis and interpretation of data: Rangel-Castilla, Russin, Zaidi, Martinez-del-Campo. Drafting the article: Russin. Critically revising the article: Albuquerque, McDougall, Spetzler. Reviewed submitted version of manuscript: Nakaji, Albuquerque, McDougall, Spetzler. Statistical analysis: Rangel-Castilla, Russin,Zaidi, Martinez-del-Campo. Administrative/ technical/material support: Rangel-Castilla, Russin, Zaidi, Park.

\section{References}

1. Aminoff MJ, Logue V: The prognosis of patients with spinal vascular malformations. Brain 97:211-218, 1974

2. Bao YH, Ling F: Classification and therapeutic modalities of spinal vascular malformations in 80 patients. Neurosurgery 40:75-81, 1997

3. Boström A, Krings T, Hans FJ, Schramm J, Thron AK, Gilsbach JM: Spinal glomus-type arteriovenous malformations: microsurgical treatment in 20 cases. Clinical article. J Neurosurg Spine 10:423-429, 2009

4. Corkill RA, Mitsos AP, Molyneux AJ: Embolization of spinal intramedullary arteriovenous malformations using the liquid embolic agent, Onyx: a single-center experience in a series of 17 patients. J Neurosurg Spine 7:478-485, 2007

5. Detweiler PW, Porter RW, Spetzler RF: Spinal arteriovenous malformations. Neurosurg Clin N Am 10:89-100, 1999

6. Djindjian R: Spinal vascular malformations. J Neurosurg 45:727-728, 1976 (Letter)

7. Fairhall JM, Reddy R, Sears W, Wenderoth JD, Stoodley MA: Successful endovascular and surgical treatment of spinal extradural metameric arteriovenous malformation. Case report. J Neurosurg Spine 13:784-788, 2010
8. Han PP, Theodore N, Porter RW, Detwiler PW, Lawton MT, Spetzler RF: Subdural hematoma from a Type I spinal arteriovenous malformation. Case report. J Neurosurg 90 (2 Suppl):255-257, 1999

9. Kalani MY, Ahmed AS, Martirosyan NL, Cronk K, Moon K, Albuquerque FC, et al: Surgical and endovascular treatment of pediatric spinal arteriovenous malformations. World Neurosurg 78:348-354, 2012

10. Kim LJ, Spetzler RF: Classification and surgical management of spinal arteriovenous lesions: arteriovenous fistulae and arteriovenous malformations. Neurosurgery 59:S3-195-S3-201, 2006

11. Lanzino G, D'Urso PI, Kallmes DF, Cloft HJ: Onyx embolization of extradural spinal arteriovenous malformations with intradural venous drainage. Neurosurgery 70:329-333, 2012

12. Linfante I, Tari Capone F, Dabus G, Gonzalez-Arias S, Lau PE, Samaniego EA: Spinal arteriovenous malformation associated with spinal metameric syndrome: a treatable cause of long-term paraplegia? Case report. J Neurosurg Spine 16:408-413, 2012

13. Lv X, Li Y, Yang X, Jiang C, Wu Z: Endovascular embolization for symptomatic perimedullary AVF and intramedullary AVM: a series and a literature review. Neuroradiology 54:349-359, 2012

14. Paraskevopoulos D, Magras I, Polyzoidis K: Spontaneous spinal epidural hematoma secondary to extradural arteriovenous malformation in a child: a case-based update. Childs Nerv Syst 29:1985-1991, 2013

15. Rangel-Castilla L, Holman PJ, Krishna C, Trask TW, Klucznik RP, Diaz OM: Spinal extradural arteriovenous fistulas: a clinical and radiological description of different types and their novel treatment with Onyx. Clinical article. J Neurosurg Spine 15:541-549, 2011

16. Rangel-Castilla L, Shah AH, Klucznik RP, Diaz OM: Preoperative Onyx embolization of hypervascular head, neck, and spinal tumors: experience with 100 consecutive cases from a single tertiary center. J Neurointerv Surg 6:51-56, 2014

17. Schievink WI, Vishteh AG, McDougall CG, Spetzler RF: Intraoperative spinal angiography. J Neurosurg 90 (1 Suppl): 48-51, 1999

18. Spetzler RF, Detwiler PW, Riina HA, Porter RW: Modified classification of spinal cord vascular lesions. J Neurosurg 96 (2 Suppl):145-156, 2002

19. Velat GJ, Chang SW, Abla AA, Albuquerque FC, McDougall CG, Spetzler RF: Microsurgical management of glomus spinal arteriovenous malformations: pial resection technique. Clinical article. J Neurosurg Spine 16:523-531, 2012

20. Vishteh AG, Zabramski JM, Spetzler RF: Patients with spinal cord cavernous malformations are at an increased risk for multiple neuraxis cavernous malformations. Neurosurgery 45: 30-33, 1999

21. Walsh DC, Zebian B, Tolias CM, Gullan RW: Intraoperative indocyanine green video-angiography as an aid to the microsurgical treatment of spinal vascular malformations. Br J Neurosurg 28:259-266, 2014

22. Wilson DA, Abla AA, Uschold TD, McDougall CG, Albuquerque FC, Spetzler RF: Multimodality treatment of conus medullaris arteriovenous malformations: 2 decades of experience with combined endovascular and microsurgical treatments. Neurosurgery 71:100-108, 2012

Manuscript submitted May 15, 2014.

Accepted July 2, 2014.

Please include this information when citing this paper: DOI: 10.3171/2014.7.FOCUS14236.

Address correspondence to: Peter Nakaji, M.D., c/o Neuroscience Publications, Barrow Neurological Institute, St. Joseph's Hospital and Medical Center, 350 W. Thomas Rd., Phoenix, AZ 85013. email: neuropub@dignityhealth.org. 